\title{
Progressive multifokale Leukenzephalopathie
}

\section{Schwerwiegende Komplikation einer Immunsuppression}

Damian Franzen, Christian Mathys, Jan Vesper, Bastian Malzkorn, Heribert Bohlen, Rüdiger J. Seitz

Unklare neurologische Krankheitserscheinungen bei immunsupprimierten Patienten können vielfältige Ursachen haben und lebensbedrohlich sein. Rasches Handeln und eine weit angelegte Diagnostik sind daher erforderlich. Im vorgestellten Fall kam es unter immunsuppressiver Therapie zu einer opportunistischen Infektion des Gehirns.

\section{Anamnese und Vorbefunde}

Grundkrankheit Sarkoidose I Erstmals vor 5 Jahren stellte sich der damals 48-jährige Patient $(72 \mathrm{~kg}, 177 \mathrm{~cm})$ zur Diagnostik in die Klinik vor, weil sich sein Allgemeinzustand verschlechtert hatte. Bis dahin war er immer gesund gewesen. Im Röntgen-Thorax sah man vereinzelte pneumonische Infiltrate und vergrößerte Lymphknoten der Lungenhili. Die Bronchoskopie bestätigte den klinischen Verdacht auf eine Sarkoidose, die bronchoalveoläre Lavage zeigte folgende Befunde:

- Lymphozytose mit 37\%,

- T-Zellen $89 \%$,

- B-Zellen 0\%,

- T4/T8-Ratio 1.7,

- Makrophagen 61\%

Eine transbronchiale Biopsie ergab einzelne nichtverkäsende Granulome. Sonographisch war eine geringe Milzvergrößerung $(13 \times 6 \mathrm{~cm})$ nachweisbar.

Remission unter Steroiden | Nach mikrobiologischem Ausschluss einer Tuberkulose wurde eine orale Steroidmedikation mit $50 \mathrm{mg} / \mathrm{d}$ Prednison eingeleitet. Nach einer rund 6-monatigen Therapie mit oralen Steroiden kam es zu einer raschen und langanhaltenden klinischen Remission, sodass die Therapie zunächst ohne weitere radiologische oder serologische Kontrollen ausgesetzt wurde.

Ausgeprägtes Rezidiv I Etwa 21/2 Jahre später trat Belastungsdyspnoe auf; ein CT-Thorax zeigte eine deutliche Progression der Lymphknotenvergrößerungen von Hilus und Mediastinum sowie eine ausgedehnte pulmonale Beteiligung mit flächenhaften Verdichtungen und multiplen Rundherden bis in die Peripherie. Unter Belastung sank die Sauerstoffsättigung von 98 auf 95\%. Die BSG war mit $22 \mathrm{~mm} / 1 \mathrm{~h}$ gering erhöht. Der lösliche Interleukin-2-Rezeptor lag mit $1852 \mathrm{U} / 1$ deutlich über der Norm (148-623 U/1).

Steroid-Nebenwirkungen | Nach ausführlicher Diskussion mit dem Patienten wurde erneut eine Therapie mit $50 \mathrm{mg} / \mathrm{d}$ Prednison eingeleitet.
- Darunter besserten sich zwar die klinischen, serologischen und radiologischen Befunde;

- dies ging jedoch einher mit einer deutlichen Erhöhung des Augeninnendrucks.

Daher wurde die Steroiddosis im Laufe eines Jahres auf $5 \mathrm{mg} / \mathrm{d}$ reduziert und parallel eine Therapie mit $100 \mathrm{mg} / \mathrm{d}$ Azathioprin eingeleitet.

- Einige Monate später kam es klinisch, laborchemisch und computertomografisch zu einem erneuten Fortschreiten der Sarkoidose.

- Dies konnte jedoch durch eine kurzzeitige Höherdosierung von Prednison auf $20 \mathrm{mg} / \mathrm{d}$ bei unveränderter Dosierung von Azathioprin erfolgreich behandelt werden.

Abb. 1 MRT-Befunde des Patienten. A (FLAIR), B (T2) und C (T1 mit Kontrastmittel): Die präoperative Untersuchung zeigt einen ausgedehnten Kontrastmittel-aufnehmenden Herdbefund rechts frontal mit ausgeprägtem perifokalen Ödem. D (T2) nach Operation: Resektionsareal (Pfeile).

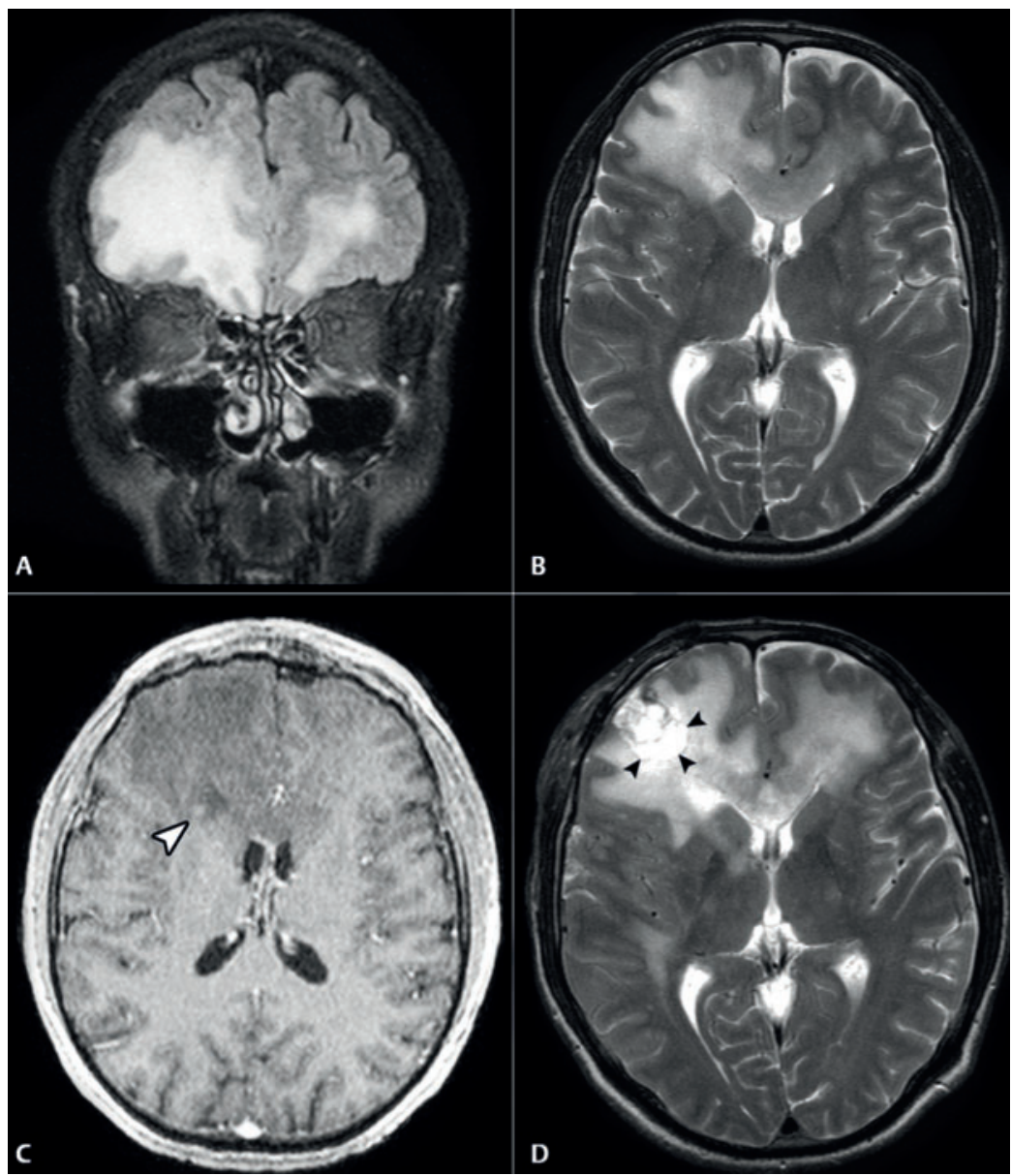


Abb. 2 Immunhistochemischer Nachweis des JC-Virus. Bei der Immunreaktion mit dem Antikörper gegen JCV-Proteine werden rundliche Zellkerne von Oligodendrozyten braun markiert, die zum Teil deutlich vergrößert sind.

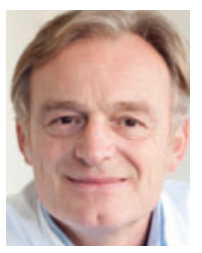

Prof. Dr. med. Damian Franzen ist Facharzt für Innere Medizin (Kardiologie, Lungen- und Bronchialheilkunde) am Prof. Franzen Institut, Köln info@damianfranzen.de

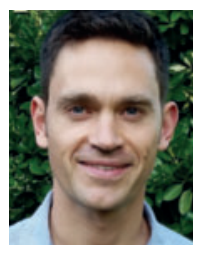

Dr. med. Christian Mathys ist Facharzt für Radiologie, Neuroradiologe und Oberarzt am Institut für Diagnostische und Interventionelle Radiologie, Universitätsklinikum Düsseldorf mathys@uni-duesseldorf.de

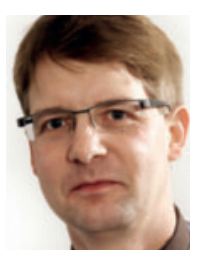

Prof. Dr. med. Jan Vesper leitet das Zentrum für Neuromodulation an der Neurochirurgischen Klinik, Universitätsklinikum Düsseldorf Jan.Vesper@ med.uni-duesseldorf.de

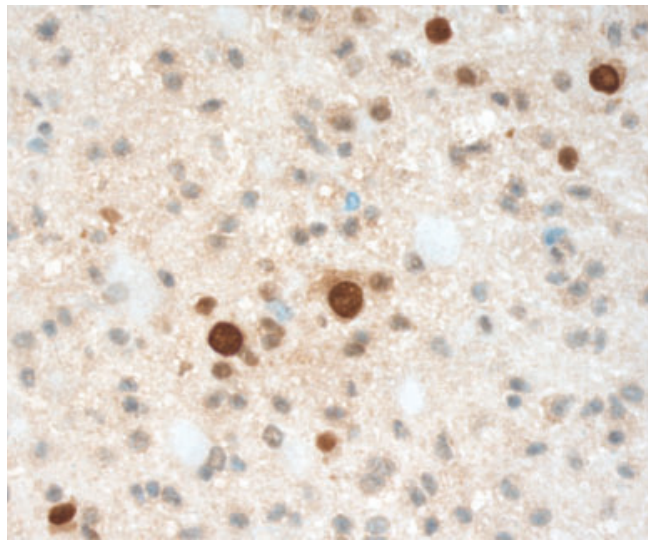

\section{Aktuelle Befunde}

Tagesmüdigkeit und Wesensveränderung | Bei einer ambulanten Kontrolluntersuchung etwa 4 Jahre nach der Erstdiagnose berichtete der Patient über Tagesmüdigkeit. Seine Ehefrau gab ferner an, ihr Ehemann habe sich in seinem Verhalten stark verändert. Das vor ihr beschriebene wesensuntypische Verhalten entsprach aus ärztlicher Sicht am ehesten einer frontalen Dissoziation. Der Patient wurde daher zur stationären Diagnostik eingewiesen.

Laborbefunde I Die BSG war mit $10 \mathrm{~mm} / 1 \mathrm{~h}$ normal, der lösliche Interleukin-2-Rezeptor war von vormals 1503 auf $1331 \mathrm{U} / \mathrm{ml}$ gering abgefallen. Die Nierenfunktion (glomeruläre Filtrationsrate $86 \mathrm{ml} / \mathrm{min}$ ) war unauffällig, auch das SerumCalcium mit 2,31 mmol/1 war normal. Im Differenzialbild fand sich eine Lymphozytopenie von

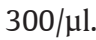

Körperliche Untersuchung | Bei stationärer Aufnahme zeigte sich ein wacher, aber desorientierter Patient mit Neigung zu Konfabulationen und Wortfindungsstörungen. Die neurologische Untersuchung ergab eine leichte Fallneigung beim Gehen nach links, ansonsten war die Befunde an Hirnnerven und der Reflexstatus unauffällig. Es bestand kein Meningismus. Der internistische Status war bis auf eine Anhidrose der Fußsohlen mit großflächigen Warzen völlig normal Diese waren bislang unbemerkt geblieben; vermutlich handelte es sich um Papillome.

Weiterführende Diagnostik | Nach klinischen Gesichtspunkten wurde zunächst an eine virale Infektion im Rahmen einer Immunsupression gedacht. Eine Magnetresonanztomographie ergab jedoch einen ausgedehnten Kontrastmittel-aufnehmenden Herdbefund rechts frontal mit ausgeprägtem perifokalen Ödem ( $\bullet$ Abb. 1 ). In erster Linie bestand der Verdacht auf einen malignen hirneigenen Tumor oder auch ein intrazerebrales Lymphom. Daher wurde initial die Indikation für eine Hirnbiopsie gestellt; sie erfolgte wenige Tage später inkl. intraoperativer Liquorentnahme.
Histologische Befunde | Da die feingeweblichen Befunde keinen Hinweis auf einen Tumor ergaben, wurde weitere Untersuchungen zur differentialdiagnostischen Abklärung einer Enzephalopathie durchgeführt. Überraschenderweise zeigten sich kräftige Markierungen mit Antikörpern gegen das JC-Virus ( Abb. 2)

- Dieser Befund ist typisch für eine progressive multifokalen Leukenzephalopathie.

Des weiteren wurde Biopsie-Material enzymatisch aufgearbeitet, um Tumorzellen zu isolieren. Nach 4 Tagen zeigte sich jedoch keine dominante Tumorzellpopulation, sondern vielmehr

- eine makrophagenartige Zellpopulation mit multiplen Inklusionskörperchen.

Eine Stimulation mit CD3- und CD28-Antikörpern führte in der Folge zu einer starken Proliferation von T-Zellen. Bei einer weiteren immunologischen Untersuchung zeigte sich eine starke, mutmaßlich Virus-spezifische Zytotoxizität gegenüber den oben beschriebenen makrophagenartigen Zellen.

Differenzialdiagnostik | Unter der noch laufenden Azathioprin-Therapie ergaben sich Hinweise für eine deutlich verminderte zelluläre Immunität mit einer Erniedrigung der CD4-T-Lymphozyten auf 147/ $\mu$ l (Norm: 420-1375) und eine CD4/CD8Ratio auf 0,4(Norm: 1-2,3). Ein HIV-Test war jedoch negativ. Ein Polymorphismus der Thiopurin-SMethyltransferase, der zu einem verzögerten Abbau von Azathioprin führt, wurde nicht analysiert.

\section{Therapie und Verlauf}

Intensivmedizinische Therapie | Die Immunsuppression mit Azathioprin wurde sofort beendet und eine Therapie

- mit Dexamethason zur Hirnödembehandlung,

- mit Mirtazapin zur Blockade serotonerger Rezeptoren und

- mit Valproat zur Anfallsprophylaxe

begonnen. Zunächst schritten die klinischen Erscheinungen eines hirnorganischen Psychosyndroms fort; es kam zu Hemiplegie und komatösen Zuständen - gleichzeitig nahmen die MR-Befunde einer Signalanhebung in rechts-temporalen und links-frontalen Hirnabschnitten zu.

Befundbesserung I Nach 3 Monaten unter stationärer Intensivpflege besserten sich die klinischen Symptome deutlich:

- Der Patient begann wieder zu sprechen.

- Die kognitiven Einschränkungen gingen zurück; lediglich das Kurzzeitgedächnis war noch beeinträchtigt.

- Dagegen bildeten sich die Lähmungserscheinungen nur zögerlich zuück.

Etwa 6 Monate nach der Diagnosestellung konnte der Patient das Bett mit Hilfe verlassen. Insgesamt besserten sich viele Symptome langsam, aber stetig. Die Fähigkeit zu sprechen verschwand wieder, nachdem zwei Grand-mal-Anfälle aufgetreten 
waren. Zusätzlich kam es trotz fehlender Immunsuppression zu bakteriellen Infekten inklusive MSRA-Besiedlung, die langwierige Antibiotika Behandlungen erforderlich machten. 12 Monate nach Diagnosestellung lebt der Patient zu Hause, ist bettlägerig und depressiv, nach internistischen Kriterien jedoch stabil.

\section{Diskussion}

Komplikation der Immunsuppression I Die bei unserem Patienten diagnostizierte progressive multifokale Leukenzephalopathie (PML) ist eine schwere Komplikation einer immunsuppressiven Therapie: Aufgrund einer verminderten zellulären Immunabwehr kommt es zu

- einer Reaktivierung des JC-Virus und

- einer Demyelinisierung im Zentralnervensystem [1].

Klinisch sind eine Vielzahl neurologischer Erscheinungen beschrieben, deren Ausprägung von der Lokalisation der zerebralen Demyelinisierungsherde abhängt. Im vorliegenden Fall stand ein hirnorganisches Psychosyndrom im Vordergrund.

Häufig tödlicher Verlauf | Über das gleichzeitige Auftreten einer PML und einer Sarkoidose gibt es mehrere Fallberichte [2,3]. Des Weiteren sind auch Fälle von PML unter immunsuppressiver Therapie anderer Erkrankungen [4] und solche unter Infektion mit dem HI-Virus bekannt [5]. Neuere Daten zeigen, dass auch unter Immunsuppression mit monoklonalen Antikörpern wie Natalizumab oder Rituximab derartige Reaktivierungen des JC-Virus beobachtet werden $[1,6]$. Viele Fälle einer progressiven multifokalen Leukenzephalopathie verlaufen innerhalb weniger Monate tödlich. Im vorliegenden Fall kam es mehrere Monate nach Absetzen von Azathioprin unter stationärer Intensivpflege zu einer deutlichen klinischen Besserung:

- Wir setzen dabei Mirtazapin ein mit dem Ziel, den Viruseintritt in Oligodendrozyten zu hemmen [7];

- ferner erfolgte eine Dexamethason-Therapie zur Hirnödem-Prophylaxe.

Andere Therapiealternativen wie beispielsweise die Gabe des Antimalariamittels Mefloquin oder Immunglobuline kamen nicht zum Einsatz [8].

Ursache der PML | Obwohl auch die Sarkoidose mit einer verminderten zellulären Immunität einhergeht, spricht der Verlauf im von uns beschriebenen Fall eher für eine Nebenwirkung der immunsuppressiven Therapie. Der bei dem Patienten zunächst nicht bemerkte großflächige Befall der Hand- und Fußflächen mit Papillomaviren mag als früher Index für die stark herabgesetzte Immunität eingestuft werden [9]. Da die Steroidmedikation durchgehend fortgesetzt wurde, scheint primär die Azathioprin-Therapie über den Weg einer verminderten zellulären Abwehr entscheidender Auslöser der Reaktivierung des JC-Polyomavirus zu sein.
Immunrekonstitution | Trotz der effektiven Immunsuppression mit Azathioprin (300 Lymphozyten pro $\mu \mathrm{l}$ ) blieb bei unserem Patienten ein inflammatorisches Immunrekonstitutionssyndrom (IRIS) aus - möglicherweise aufgrund der begonnenen Dexamethasongabe. Das IRIS ist eine häufige und gefürchtete Komplikation nach Beenden einer immunsuppressiven Behandlung [10]. Ob die Rückbildung der klinischen Symptome im geschilderten Fall Folge der Therapie oder spontaner Erholung dank wiederhergestellter zellulärer Immunität war, bleibt offen.

\section{Konsequenz für Klinik und Praxis}

- Bei unerwarteten neurologischen Ausfallserscheinungen unter immunsuppressiver Therapie sollte an eine progressive multifokale Leukenzephalopathie (PML) gedacht werden.

- Die Diagnose lässt sich durch JC-Virusnachweis im Liquor oder - wie im geschilderten Fall - im Hirngewebe sichern.

- Frühzeitiges Absetzen der Immunsuppression und intensivmedizinische Therapie können den sonst meist letalen Verlauf in Einzelfällen aufhalten.

\section{Literatur}

1 Bellizzi A, Anzivino E, Rodio DM et al. New insights on human poliomavirus JC and pathogenesis of progressive multifocal leukencephalopathy. Clini Dev Immunol 2013; 2013: 839719

2 Marriott PJ, O’Brien MD, Mackensie J. Progressive multifocal leukoencephalopathy: remission with cytarabine. Neurol Neurosurg Psychiatr 1975; 38: 205-209

3 Rosenheim MA, Uphoff DF. The association of progressive multifocal leukoencephalopathy and sarcoidosis. Chest 1983; 83: 572-575

4 Chihara D, Takeoka T, Shirase T et al. Progressive multifocal leukencephalopathy in myelodysplastic syndrome involving pure red cell aplasia. Intern Med 2010; 49: 2347-2352

5 Brew BJ, Davies NWS, Cinque $P$ et al. Progressive multifocal leukoencephalopathy and other forms of JC virus disease. Nat Rev Neurol 210; 6: 667-679

6 Küllmer A, Herrmann G, Riecken B. Rasch fortschreitender geistiger und körperlicher Verfall bei einem Patienten mit B-Zell-Non-HodgkinLymphom unter Therapie mit Rituximab und Bendamustin. Dtsch Med Wochenschr 2013; 138 : 1355-1359

7 Cettomai D, McArthur JC. Mirtazapine use in human immunodeficiency virus-infected patients with progressive multifocal leukoencephalopathy. Arch Neurol 2009; 66: 255-258

8 Kalisch A, Erbguth WM, Birkmann J. Progressive multifocal leukencepalopathy in patients with a hematological malignancy. Review of therapeutic options. Chemotherapy 2014; 60: 47-53

9 Wieland U, Kreuter A, Pfister H. Human papillomavirus and immunosuppression. Curr Probl Dermatol 2014; 45: 154-165

10 Martin-Blondel G, Delobel P, Blancher A et al. Pathogenesis of the immune reconstitution inflammatory syndrome affecting the central nervous system in patients infected with HIV. Brain 2011; 134: 928-946

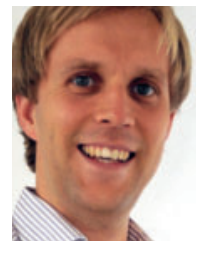

Dr. med. Bastian Malzkorn ist Mitarbeiter des Instituts für Neuropathologie am Universitätsklinikum,

Düsseldorf

bastian.malzkorn@ uni-duesseldorf.de

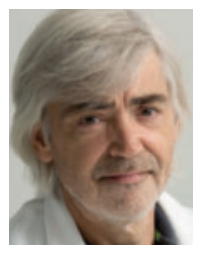

Prof. Dr. med. Heribert Bohlen, PhD Chief Executive Officer Axiogenesis AG, Köln bohlen@axiogenesis.com

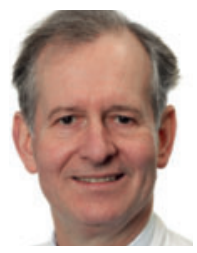

Prof. Dr. med. Rüdiger J. Seitz Leitung Neurologie, Zentrum für Neurologie und Neuropsychiatrie,

LVR-Klinikum Düsseldorf, sowie Neurologische Klinik, Universitätsklinikum

Düsseldorf

seitz@neurologie.uniduesseldorf.de

\section{Interessenkonflikt}

Die Autoren erklären, dass kein Interessenkonflikt besteht.

DOI 10.1055/s-0041-103278

Dtsch Med Wochenschr 2015; 140: 1291-1293

(c) Georg Thieme Verlag KG . Stuttgart · New York . ISSN 0012-0472 